\title{
Representação do efeito de inibição enzimática reversível para o modelo cinético de Michaelis-Menten no estado transiente
}

\author{
Representation of the reversible enzymatic inhibition effect for the kinetic \\ model of Michelis-Menten in transient state
}

\section{Autores | Authors}

\section{*André Rosa MARTINS}

Instituto Federal de Educação, Ciência e Tecnologia do Rio Grande do Sul (IFRS) Campus Porto Alegre Rua Cel. Vicente, 281, Bairro Centro CEP: $90.030-041$ Porto Alegre/RS - Brasil e-mail: andre.martins@poa.ifrs.edu.br

*Autor Correspondente / Corresponding Author

Recebido: Set. 23, 2014

Aprovado: Ago. 05, 2015

\section{Resumo}

Os processos enzimáticos que seguem o modelo cinético de MichaelisMenten foram estudados a partir de diferentes propostas para descrever a etapa de inibição reversível. As propostas de inibição foram comparadas a partir de um processo genérico, onde as constantes cinéticas receberam valores unitários e o valor numérico da concentração de substrato foi dez (10) vezes superior ao valor numérico da concentração de enzima. Para cada proposta de modelo de inibição foram obtidas soluções numéricas a partir de sistema não linear de equações diferenciais ordinárias, gerando gráficos que apresentaram, separadamente, a variação das concentrações da enzima, dos complexos enzimáticos, do substrato e do produto da reação. Foi obtido um modelo, dentre as propostas avaliadas, com desempenho indicando comportamento similar ao verificado no modelo clássico de Michaelis-Menten, onde o complexo de reação é rapidamente formado e, ao longo do processo, decai até tender a zero. Em contrapartida, diferentemente do modelo clássico, na nova proposta de modelo o efeito de inibição começa em zero e, ao longo do processo, tende ao valor nominal da concentração inicial da enzima. Tais respostas mostraram-se válidas para valores distintos de concentração de enzima e de tempo de processo, mostrando robustez e indicando uma tendência do somatório do substrato e do produto atingir o valor nominal da concentração inicial do substrato ao longo do tempo de processamento.

Palavras-chave: Catálise enzimática; Modelo cinético; Modelo de MichaelisMenten.

\section{Summary}

Enzymatic processes that follow the kinetic model of Micahelis-Merten had been studied taking as reference different proposals to describe the reversible inhibition phase. Inhibition proposals were compared based on a generic process. In this process, the kinetic constants received unitary values and the numeric value of the substrate concentration was ten (10) times higher than the numeric value of enzyme concentration. Numeric solutions based on a non-linear system of ordinary differential equations were obtained for each proposal of the inhibition model, resulting in a graphic representing, separately, the variation of enzyme concentrations, enzymatic complexes, substrate and product reaction. One model was obtained, among the evaluated proposals, with performance indicating behavior similar to the classical Michaelis-Menten model, where the reaction complex is rapidly formed and, along the process, decay tending to zero. However, differently from the classic model, in the proposed new model the inhibition process starts at zero and, during the process, tends to the nominal value of the initial enzymatic concentration. Such responses were shown to be valid for different values of enzyme concentration and processing time, showing robustness and indicating that the sum of the substrate and the product tends to reach the nominal value of the initial concentration of the substrate along the processing time.

Key words: Enzymatic catalysis; Kinetic model; Michaelis-Menten model. 


\section{Introdução}

Na modelagem matemática para cinética aplicada, a maioria das reações enzimáticas parte-se da hipótese de Michaelis-Menten, que descreve a velocidade de reação na condição em que a concentração de substrato é superior à concentração da enzima e a soma das concentrações da enzima e dos complexos enzimáticos formados se mantém constante ao longo do tempo de processamento (JOHNSON e GOODY, 2011).

Existe carência de dados na literatura no que se refere à condição em que a etapa de inibição se desenvolve. O modelo clássico de Michaelis-Menten não contempla a inibição, muito embora a ampla maioria dos processos enzimáticos não propicie conversão de 100\% do substrato, indicando que existe um ponto em que, para uma dada condição, o processo é interrompido (CHAUDHURY e IGOSHIN, 2010).

A literatura na área de bioquímica aponta quatro (4) possíveis mecanismos de inibição enzimática reversível, quais sejam: competitiva; não competitiva; mista e incompetitiva (IUB, 1982).

Os trabalhos disponíveis na literatura, via de regra, tratam a resolução da etapa de inibição a partir do pressuposto do estado estacionário, apontando um presumido equilíbrio entre a formação e a dissociação do complexo enzima substrato (TZAFRIRI e EDELMAN, 2007; STOLERIU et al., 2004; RECHT et al., 2009; BAKALIS et al., 2012).

Os inibidores são tradicionalmente caracterizados a partir do pressuposto de equilíbrio, no estado estacionário, entre o complexo enzimático de reação e o substrato. Esse pressuposto seria válido apenas para enzimas muito ineficientes (FANGE et al., 2011).

O presente trabalho se propõe a avaliar as diferentes possibilidades de inibição do processo representado pela cinética clássica de MichaelisMenten e sugerir novas representações para a etapa de inibição reversível da catálise em modelos matemáticos adequados à representação no estado transiente. Para tal, é utilizado o recurso de definir um processo genérico onde o valor nominal da concentração do substrato é superior em dez (10) vezes ao valor nominal da concentração da enzima. Todas as constantes cinéticas, nesse modelo pictórico, assumem, inicialmente, o valor unitário. A solução numérica dos sistemas não lineares de equações diferenciais ordinárias (EDOs) foi obtida a partir de uma rotina elaborada no Matlab ${ }^{\circledR} \mathrm{Com}$ aplicação do método de Runge-Kutta de $4^{a}$ ordem. As respostas são apresentadas em figuras com dois gráficos, mostrando separadamente a variação das concentrações da enzima e dos complexos enzimáticos e, de outra parte, a variação das concentrações do substrato e do(s) produto(s).

\subsection{Revisão dos modelos da literatura}

Para proceder à avaliação das diferentes perspectivas de inibição reversível, faz-se necessário, inicialmente, uma abordagem sobre a cinética clássica de Michaelis-Menten aplicada a um processo de catálise genérico, conforme o Esquema 1, onde as constantes $\mathrm{k}_{1}, \mathrm{k}_{-1}, \mathrm{k}_{2}, \mathrm{k}_{-2}$ e $\mathrm{k}_{3}$ são as constantes cinéticas dessas reações. Os demais componentes são: a concentração de enzima $\mathrm{E}$; a concentração de substrato $\mathrm{S}$; a concentração do complexo enzima substrato ES; a concentração do complexo enzima produto EP; a concentração do produto P.

$\mathrm{Na}$ modelagem matemática das reações são obtidas as variações do substrato, da enzima, do produto e dos complexos enzimáticos, conforme segue:

Esquema 1-A (Equações 01 a 04)

$$
\begin{aligned}
& \frac{d E}{d t}=k_{-1} E S-k_{1}(E \cdot S)+k_{2} E S \\
& \frac{d E S}{d t}=k_{1}(E \cdot S)-k_{-1} E S-k_{2} E S \\
& \frac{d S}{d t}=k_{-1} E S-k_{1}(E \cdot S) \\
& \frac{d P}{d t}=k_{2} E S
\end{aligned}
$$

Esquema 1-B (Equações 05 a 09)

$$
\frac{d E}{d t}=k_{-1} E S-k_{1}(E \cdot S)+k_{3} E P
$$

$\frac{d E P}{d t}=k_{2} E S-k_{-2} E P-k_{3} E P$

$$
\frac{d E S}{d t}=k_{1}(E \cdot S)-k_{-1} E S+k_{-2} E P-k_{2} E S
$$

$$
\frac{d S}{d t}=k_{-1} E S-k_{1}(E \cdot S)
$$

$$
\frac{d P}{d t}=k_{3} E P
$$

$$
\begin{aligned}
& \text { (A) } E+S \stackrel{\overrightarrow{k_{1}}}{\longrightarrow} E S \stackrel{k_{-1}}{\longrightarrow} E+P \\
& \text { (B) } E+S \underset{\substack{k_{-1} \\
\longleftarrow}}{\stackrel{\overrightarrow{k_{1}}}{\longrightarrow}} E S \underset{\substack{k_{-2} \\
\leftarrow}}{\stackrel{\overrightarrow{k_{2}}}{\longrightarrow}} E P \stackrel{k_{3}}{\longrightarrow} E+P
\end{aligned}
$$

Esquema 1. Modelos cinéticos clássicos de Michaelis-Menten. 
Para a solução das equações diferenciais ordinárias dos sistemas não lineares expressos anteriormente, a principal exigência diz respeito à estrita observação à Lei de Conservação de Massas para a enzima e o(s) complexo(s) (Equação 10), através das expressões a seguir:

1-A) $\frac{d E}{d t}+\frac{d E S}{d t}=\frac{d}{d t}(E+E S)=0$
$1-B) \frac{d E}{d t}+\frac{d E P}{d t}+\frac{d E S}{d t}=\frac{d}{d t}(E+E P+E S)=0$

As soluções numéricas desses modelos, aplicada para o processo genérico sugerido, são mostradas nas Figuras 1 e 2, respectivamente para as alternativas "A" e "B" do Esquema 1, com concentração de substrato e de enzima com unidade hipotética $(X)$, que representa

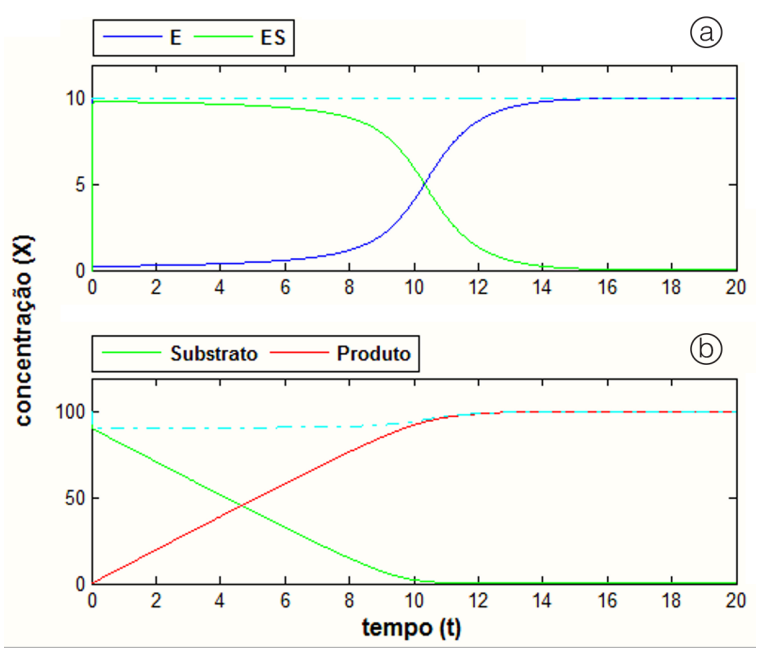

Figura 1. Resposta do modelo cinético clássico de MichaelisMenten referente ao Esquema 1-A.

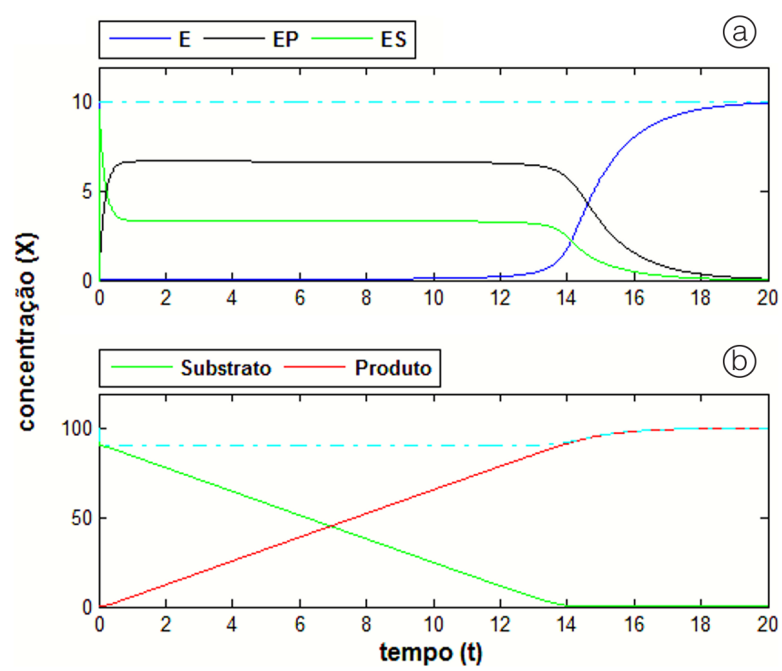

Figura 2. Resposta do modelo cinético clássico de MichaelisMenten referente ao Esquema 1-B. uma relação entre massa e volume, e com o tempo de processo com unidade hipotética (t). Na Figura 1 todas as constantes cinéticas assumem $\mathrm{o}$ valor unitário e na Figura 2 fez-se $\mathrm{k}_{1}=\mathrm{k}_{2}=4$ e as demais constantes foram mantidas com valor unitário, para manter o mesmo tempo de processo em ambas as respostas.

Os gráficos das Figuras 1a e 2a apresentam a variação da enzima e do(s) complexo(s) enzimático(s) e, nas linhas pontilhadas, a soma das contribuições dessas variáveis. Os gráficos das Figuras 1-b e 2-b apresentam a variação do substrato e do produto e, nas linhas pontilhadas, a soma da contribuição desses dois componentes. As linhas pontilhadas das Figuras 1a e 2a se mantêm sempre no valor inicial da enzima, o que condiz com a Lei de Conservação de Massas.

Nas linhas pontilhadas das Figuras $1 \mathrm{~b}$ e $2 \mathrm{~b}$ observa-se uma queda no início e uma convergência posterior ao valor nominal do substrato adicionado. Esta queda inicial está vinculada à relação entre enzima e substrato e será igual ao valor da fração entre esses componentes. No exemplo mostrado essa fração é igual a 10/100, ou seja, representa uma queda inicial de 10\% no somatório substrato e produto, quando do início do processo, tendendo ao valor da concentração inicial do substrato ao final do processo.

O complexo de reação ES (Equação 11), em ambas as alternativas, é formado imediatamente no início da reação e, após, decresce desde um valor inicial muito próximo ao valor nominal da enzima até zero. Numa parte expressiva do tempo de processo a variação da concentração desse complexo é pequena, indicando a possibilidade de considerar-se o estado como 'quase estacionário', ou seja:

$$
\frac{\mathrm{dES}}{\mathrm{dt}} \cong 0
$$

\subsection{Revisão dos modelos de inibição}

Uma representação genérica das diferentes possibilidades de inibição enzimática reversível é mostrada no Esquema 2, conforme proposta modificada por Schenker e Baici (2009) para o mecanismo proposto por Botts e Morales (1953).

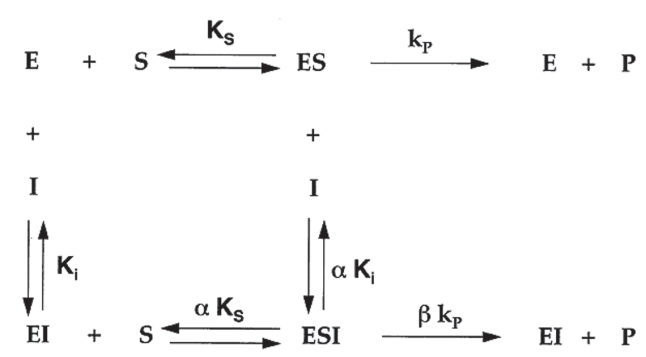

Esquema 2. Mecanismo modificado para inibição do modelo de Michaelis-Menten. Fonte: Schenker e Baici (2009). 
Nessa proposta aparecem dois novos complexos enzimáticos: El representa o complexo de inibição e ESI é o complexo intermediário de inibição. Nesse mecanismo geral aparece, também, a representação de uma substância I que será responsável pela inibição.

Em alguns trabalhos da literatura, a substância que propicia o efeito de inibição é representada por $\mathrm{R}$, sendo tratada como uma molécula modificada no processo enzimático (JIA et al., 2012).

No Esquema 2 (Equações 12 a 18), o coeficiente $\alpha$ define a proporção entre as formas competitiva $e$ incompetitiva de inibição e $\beta$ é um fator de multiplicação da constante de catálise. A representação para o sistema de EDOs referente ao mecanismo geral do Esquema 2 é mostrada a seguir:

$$
\begin{aligned}
& \frac{d E}{d t}=k_{S} E S-k_{S}(E \cdot S)+k_{p} E S+k_{i}(E I \cdot S)-k_{i}(E \cdot S \cdot l) \\
& \frac{d E S}{d t}=k_{S}(E \cdot S)-k_{S} E S-k_{p} E S+\alpha k_{i} E S I-\alpha k_{i}(E S \cdot l)
\end{aligned}
$$

$$
\frac{d E S I}{d t}=\alpha k_{i}(E S \cdot I)-\alpha k_{i} E S I+\alpha k_{S}(E I \cdot S)-\alpha k_{S} E S I-\beta k_{p} E S I
$$

$\frac{d E I}{d t}=k_{i}(E \cdot S \cdot I)-k_{i}(E I \cdot S)+\alpha k_{S} E S I-\alpha k_{S}(E I \cdot S)+\beta k_{p} E S I$

$\frac{d S}{d t}=k_{S} E S-k_{S}(E \cdot S)+\alpha k_{S} E S I-\alpha k_{S}(E I \cdot S)$

$$
\frac{d P}{d t}=k_{p} E S+\beta k_{p} E S I
$$

$$
\frac{d l}{d t}=k_{i}(E I \cdot S)-k_{i}(E \cdot S \cdot I)+\alpha k_{i} E S I-\alpha k_{i}(E S \cdot I)
$$

A Figura 3 apresenta a solução numérica do sistema de equações diferenciais ordinárias anteriores, a partir do modelo genérico e com valores unitários para os fatores $\alpha$ e $\beta$.

O que se observa nessa resposta é que o comportamento da enzima e de seus complexos é similar ao do modelo cinético clássico de Michaelis-Menten sem inibição. A resposta da Figura 3-a indica que os complexos ESI e El permanecem com valor numérico igual a zero ao longo de todo o tempo de processamento. Por consequência, na Figura 3-b, a concentração da substância de inibição, I, também se manterá com valor numérico igual a zero.

A resposta observada nesse mecanismo genérico se repetirá caso se estude, individualmente, as propostas de inibição competitiva, não-competitiva, incompetitiva ou mista.

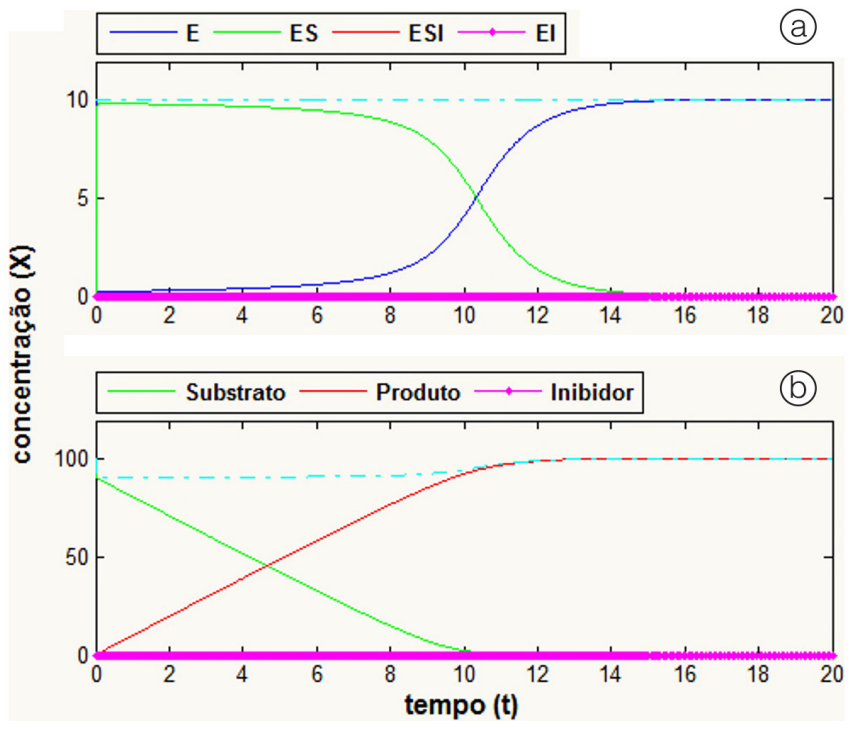

Figura 3. Resposta ao mecanismo de inibição do modelo de Michaelis-Menten.

Em realidade a resposta será numericamente idêntica àquela mostrada nos gráficos da Figura 3, tanto para a variação da enzima e seus complexos quanto para a variação do substrato e produto, ainda que sejam zerados os valores numéricos de todas as constantes cinéticas associadas ao efeito de inibição.

Tal comportamento indica que o modelo do Esquema 2 apresenta o mesmo perfil de resposta tanto para reações enzimáticas com inibição reversível quanto para reações enzimáticas sem inibição.

\section{Proposta de novo modelo}

Para a elaboração de um modelo visando representar o efeito de inibição reversível para a cinética de Michaelis-Menten faz-se necessário, inicialmente, estabelecer algumas premissas:

a) O modelo deve seguir estritamente a Lei de Conservação de Massas, no que se refere à variação do somatório da enzima e de seus complexos, ao longo de todo o tempo de processamento e de forma contínua.

b) O modelo deve convergir o somatório do substrato e produto ao valor nominal do substrato adicionado, em um tempo suficientemente elevado e com o menor erro possível, para garantir estabilidade ao processo.

c) O modelo deve ser robusto e permitir a variação na relação enzima/substrato em um tempo suficientemente elevado, garantindo estabilidade ao processo. 
d) O modelo deve propiciar um desempenho do complexo de reação, ES, com uma alta taxa de formação no início da reação e com a redução de sua concentração ao longo do tempo, tendendo a zero ao final do processamento.

e) $\mathrm{O}$ modelo deve propiciar um desempenho do complexo de inibição, El, com crescimento desde zero e tendendo ao valor nominal da enzima ao final do processamento.

O Esquema 3 mostra um modelo com três diferentes produtos de reação $\left(P_{1}, P_{2}\right.$ e $\left.P_{3}\right)$, com a formação de um único complexo intermediário de reação $\left(E P_{1}\right)$ e com o efeito de inibição associado apenas a $P_{1} e$ $\mathrm{P}_{2}$, em um típico exemplo de ação de uma hidrolase que possua capacidade tanto para hidrolisar um sacarídeo quanto para sintetizar oligossacarídeos.

Presume-se, para efeito de resolução desse modelo hipotético, que $S$ é um dissacarídeo e que, portanto, $\mathrm{P}_{1}$ e $\mathrm{P}_{2}$ são monossacarídeos que possuem a mesma massa molecular.

O mecanismo geral mostrado no Esquema 3 (Equações 19 a 26) resulta em um sistema não linear com oito (8) equações diferenciais ordinárias e onze (11) constantes cinéticas.

$$
\begin{aligned}
& \frac{d E}{d t}=k_{-1} E S-k_{1}(E \cdot S)+k_{3}\left(E P_{1} \cdot S\right)-k_{-3}\left(E \cdot P_{3}\right)+ \\
& k_{4} E P_{1}-k_{-4}\left(E \cdot P_{1}\right)+k_{-6} E I-k_{6}\left(E \cdot P_{1} \cdot P_{2}\right) \\
& \frac{d E P_{1}}{d t}=k_{2} E S+k_{-3}\left(E \cdot P_{3}\right)-k_{3}\left(E P_{1} \cdot S\right)+ \\
& k_{-4}\left(E \cdot P_{1}\right)-k_{4} E P_{1}+k_{-5} E I-k_{5}\left(E P_{1} \cdot P_{2}\right) \\
& \frac{d E S}{d t}=k_{1}(E \cdot S)-k_{-1} E S-k_{2} E S
\end{aligned}
$$

$$
\begin{aligned}
& E+S \stackrel{\vec{k}_{1}}{\longrightarrow} E S \stackrel{k_{2}}{\longrightarrow} E P_{1}+P_{2} \\
& E P_{1}+S \underset{\substack{k_{-3}}}{\stackrel{\overrightarrow{k_{3}}}{\longrightarrow}} E+P_{3} \\
& E P_{1} \underset{\substack{k_{-4} \\
\leftarrow}}{\stackrel{k_{4}}{\longrightarrow}} E+P_{1} \\
& E P_{1}+P_{2} \underset{k_{-5}}{\stackrel{\overrightarrow{k_{5}}}{\longrightarrow}} E I \stackrel{\overrightarrow{k_{-6}}}{\stackrel{k_{6}}{\longrightarrow}} E+P_{1}+P_{2}
\end{aligned}
$$

$$
\frac{\mathrm{dEI}}{\mathrm{dt}} \cong-\frac{\mathrm{dES}}{\mathrm{dt}}
$$

(etapa inicial de reação)

(etapa intermediária de reação)

(etapa final de reação)

(etapa de inibição)

Esquema 3. Proposta de inibição pelo produto para um modelo hipotético. 
A proposta de modelo do presente trabalho pressupõe o mecanismo de inibição pelo produto para expressar a etapa final da catálise, postulando que o efeito de inibição ocorre sobre o complexo de reação (ES), formando um complexo de inibição (EI). O efeito de inibição será tanto do tipo competitivo para $P_{1} e$ $\mathrm{P}_{2}$ quanto do tipo incompetitivo para $\mathrm{P}_{2}$, respeitada a estequiometria da reação, conforme proposta de Martins e Oliveira (2014).

Na Figura 4-b observa-se um erro no somatório dos produtos e substrato ao final do processo, indicando que não ocorre conservação de massa do modelo para o ajuste das constantes cinéticas apresentado na resposta da Figura 4. O Apêndice mostra um arquivo para solução do modelo desenvolvido no presente trabalho.

$\mathrm{Na}$ Figura 5 fez-se $k_{4}=1,34355$, mantendo as demais constantes cinéticas com os mesmos valores numéricos da resposta da Figura 4 . O tempo de processo foi ampliado em cinco (5) vezes e a concentração de enzima foi reduzida em dez (10) vezes $\left(E_{0}=1\right)$, para avaliar a robustez do modelo.

Na Figura 5 é possível observar que a linha que representa a Conservação de Massa do Modelo (CMM) tende para o mesmo valor da concentração inicial do substrato, ao final do processamento.

A conservação de massa do modelo (CMM) e o erro ao final do processo $\left(E_{f}(\%)\right)$ (mostrados na Equação 29 e 30) foram calculados a partir das expressões a seguir, onde $\mathrm{S}_{0}$ é a concentração inicial do substrato, $y_{i}$ representa os valores de concentração de cada variável ao longo do tempo e $y_{i}(n+1)$ representa o valor da concentração de cada variável ao final do processo. A solução foi obtida pelo método de Runge-Kutta de ordem 4 e passo $\left(\mathrm{h}\right.$ ) de $1.10^{-3}$.

$$
\begin{aligned}
& C M M=\sum_{i=1}^{v}\left(y_{i}\right) \\
& E_{f}(\%)=\left[1-\frac{\sum_{i=1}^{v}\left(y_{i}(n+1)\right)}{S_{0}}\right] * 100
\end{aligned}
$$

Na Figura 6 retomam-se os parâmetros anteriores de processo, com o valor numérico da concentração inicial do substrato igual a 100, o valor numérico da concentração de enzima igual a 10 e o valor numérico do tempo de processo igual a 20.

Os valores numéricos das constantes cinéticas para a resposta da Figura 6 foram os seguintes: $\mathrm{k}_{1}=\mathrm{k}_{2}=\mathrm{k}_{-3}=10 ; \mathrm{k}_{4}=5 ; \mathrm{k}_{-4}=4,98658$, com as demais constantes unitárias.

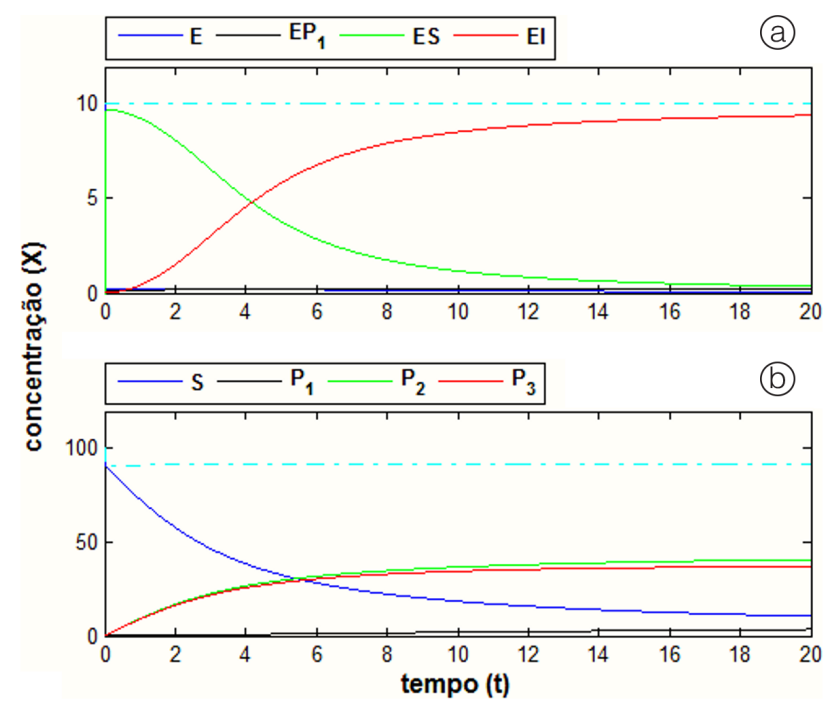

Figura 4. Resposta ao mecanismo genérico do modelo do presente trabalho.

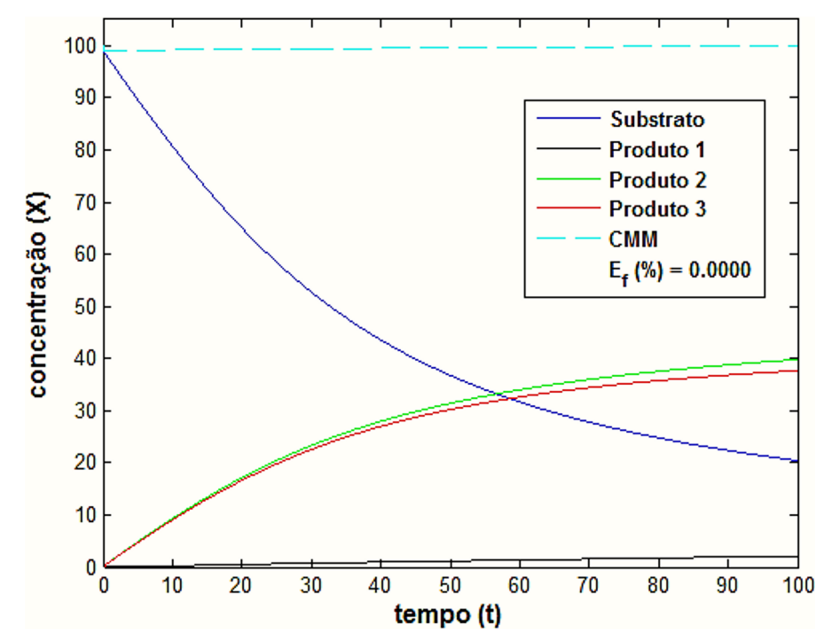

Figura 5. Resposta ao mecanismo do presente trabalho $\left(S_{0}=100 ; E_{0}=1 ; t=100\right)$.

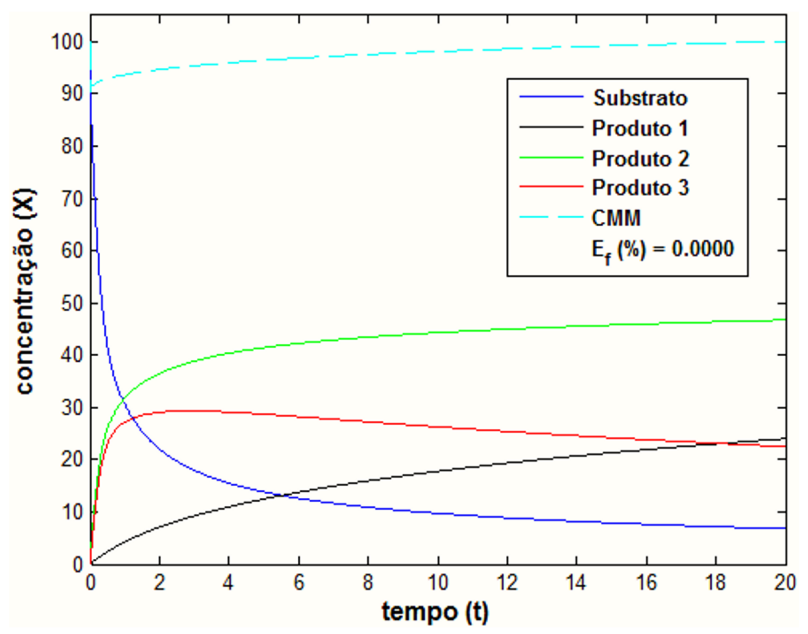

Figura 6. Resposta ao mecanismo do presente trabalho $\left(S_{0}=100 ; E_{0}=10 ; t=20\right)$. 
Os resultados mostrados nas Figuras 5 e 6 indicam que pode ser obtido um ajuste, a partir de um conjunto de valores numéricos de constantes cinéticas, que minimize o erro ao final do processo para reações enzimáticas do modelo do presente trabalho.

O ajuste do modelo a valores experimentais pode ser obtido a partir da identificação de um conjunto de constantes cinéticas que minimize o erro ao final do processo e aproxime os valores experimentais da resposta do modelo, conforme demonstrado por Martins e Lisbôa (2015), em um trabalho que avaliou a hidrólise da lactose e a síntese de galacto-oligossacarídeos.

\section{Conclusão}

Os mecanismos de inibição reversível nos processos de catálise enzimática que seguem o modelo clássico de Michaelis-Menten ainda não estão suficientemente esclarecidos.

No presente trabalho a etapa de inibição do processo enzimático, representado pelo modelo cinético de Michaelis-Menten, teve seu comportamento descrito ao aplicar-se proposta de inibição reversível por efeito do incremento da concentração do(s) produto(s), em solução numérica de um modelo genérico.

Em processos que gerem mais de um produto da catálise, o erro percentual pode ser minimizado com um adequado ajuste das taxas de velocidade de reação. A abordagem e a construção de novos modelos devem ser diferenciadas e aplicadas para cada situação específica, nos casos de catálises enzimáticas que gerem diferentes produtos de reação.

\section{Referências}

BAKALIS, E.; KOSMAS, M.; PAPAMICHAEL, E. M. Perturbation theory in the catalytic rate constant of the Henri-MichaelisMenten enzymatic reaction. Bulletin of Mathematical Biology, New York, v. 74, n. 11, p. 2535-2546, 2012. PMid:22926529.

BOTTS, J.; MORALES, M. Analytical description of the effects of modifiers and of enzyme multivalency upon the steady state catalyzed reaction rate. Transactions of the Faraday Society, London, v. 49, p. 696-707, 1953. http://dx.doi.org/10.1039/ tf9534900696. <

CHAUDHURY, S.; IGOSHIN, O. A. Dynamic disorder in quasiequilibrium enzymatic systems. PLoS One, San Francisco, v. 5, n. 8, p. e12364, 2010. http://dx.doi.org/10.1371/journal. pone.0012364. PMid:20808776.

FANGE, D.; LOVMAR, M.; PAVLOV, M. Y.; EHRENBERG, M. Identification of enzyme inhibitory mechanisms from steady- state kinetics. Biochimie, Paris, v. 93, n. 9, p. 1623-1629, 2011. http://dx.doi.org/10.1016/j.biochi.2011.05.031. PMid:21689716.

INTERNATIONAL UNION OF BIOCHEMISTRY - IUB. Nomenclature Committee of the International Union of Biochemistry (NC-IUB). Symbolism and terminology in enzyme kinetics. Recommendations 1981. European Journal of Biochemistry, England, v. 128, n. 2-3, p. 281-291, 1982. PMid:7151780.

JIA, C.; LIU, X.-F.; QIAN, M.-P.; JIANG, D.-Q.; ZHANG, Y.-P. Kinetic behavior of the general modifier mechanism of Botts and Morales with non-equilibrium binding. Journal of Theoretical Biology, Amsterdam, v. 296, n. 296, p. 13-20, 2012. http://dx.doi. org/10.1016/j.jtbi.2011.11.006. PMid:22100501.

JOHNSON, K. A.; GOODY, R. S. The original Michaelis constant: translation of the 1913 Michaelis-Menten paper. Biochemistry, Washington, v. 50, n. 39, p. 8264-8269, 2011. http://dx.doi. org/10.1021/bi201284u. PMid:21888353.

MARTINS, A. R.; LISBÔA, C. R. Mathematical model for the conversion of lactose and synthesis of galacto-oligosacharides (GOS) with simultaneous reversible inhibition by glucose and galactose. International Journal of Engineering Research and Technology, Gujarat, v. 4, n. 4, p. 299-305, 2015.

MARTINS, A. R.; OLIVEIRA, L. Michaelis-Menten kinetics in transient state: proposal for reversible inhibition model and its application on enzymatic hydrolysis of disaccharides. International Journal of Engineering Research and Applications, Ghaziabad, v. 4, n. 11, p. 101-112, 2014.

RECHT, M. I.; TORRES, F. E.; DE BRUYKER, D.; BELL, A. G.; KLUMPP, M.; BRUCE, R. H. Measurement of enzyme kinetics and inhibitor constants using enthalpy arrays. Analytical Biochemistry, Amsterdam, v. 388, n. 2, p. 204-212, 2009. http:// dx.doi.org/10.1016/j.ab.2009.02.028. PMid:19250916.

SCHENKER, P.; BAICI, A. Simultaneous interaction of enzymes with two modifiers: reappraisal of kinetic models and new paradigms. Journal of Theoretical Biology, Amsterdam, v. 261, n. 2, p. 318-329, 2009. http://dx.doi.org/10.1016/j. jtbi.2009.07.033. PMid:19660476.

STOLERIU, I.; DAVIDSON, F. A.; LIU, J. L. Quasi-steady state assumptions for non-isolated enzyme-catalysed reactions. Journal of Mathematical Biology, Heidelberg, v. 48, n. 1, p. 82-104, 2004. http://dx.doi.org/10.1007/s00285-003-0225-7. PMid:14685773.

TZAFRIRI, A. R.; EDELMAN, E. R. Quasi-steady-state kinetics at enzyme and substrate concentrations in excess of the MichaelisMenten constant. Journal of Theoretical Biology, London, v. 245 , n. 4, p. 737-748, 2007. http://dx.doi.org/10.1016/j. jtbi.2006.12.005. PMid:17234216. 

MARTINS, A. R.

Apêndice. Rotina de solução.

O apêndice do presente trabalho mostra um arquivo para solução do modelo desenvolvido, aplicada à rotina ODE45 do Matlab ${ }^{\circledR}$.

$\%$

\% Rotina no Matlab ${ }^{\circledR}$ para gerar a função vetorial F(y) para a resposta apresentada na Figura 4 $\%$

function Figura 4

options=odeset('Abstol',1e-6,'Reltol',1e-6);

t0 =0;

$\mathrm{tf}=20$;

SO =100;

$\mathrm{EO}=10$;

[t y]=ode45(@fdo,[t0 tf],[E0 000 S0 000 E0 S0],options);

subplot $(2,1,1)$

plot(t,y(:,1),'blue',t,y(:,2), 'black',t,y(:,3),'green',t,y(:,4),'red',t,y(:,9),'-.cyan');

ylabel('concentração (X)');

xlabel('tempo(t)');

$\operatorname{lgnd1}=$ ' $\mathrm{E}$ ';

Ignd2 = 'EP_1';

$\operatorname{lgnd} 3=$ 'ES';

lgnd4 = 'El';

legend(Ignd1,Ignd2,Ignd3,Ignd4);

axis([0 tf $0 \mathrm{E0}+1.9])$;

subplot $(2,1,2)$

plot(t,y(:,5), 'blue',t,y(:,6),'black',t,y(:,7),'green',t,y(:,8),'red',t,y(:,10),'-.cyan');

ylabel('concentração(X)');

xlabel('tempo (t)');

$\operatorname{lgnd1}=$ ' $\mathrm{S}$ ';

lgnd $2=$ 'P_1';

Ignd3 = 'P_2';

lgnd4 = 'P_3';

legend(Ignd1, Ignd2,Ignd3,Ignd4);

axis([0 tf $0 \mathrm{SO}+19])$; 


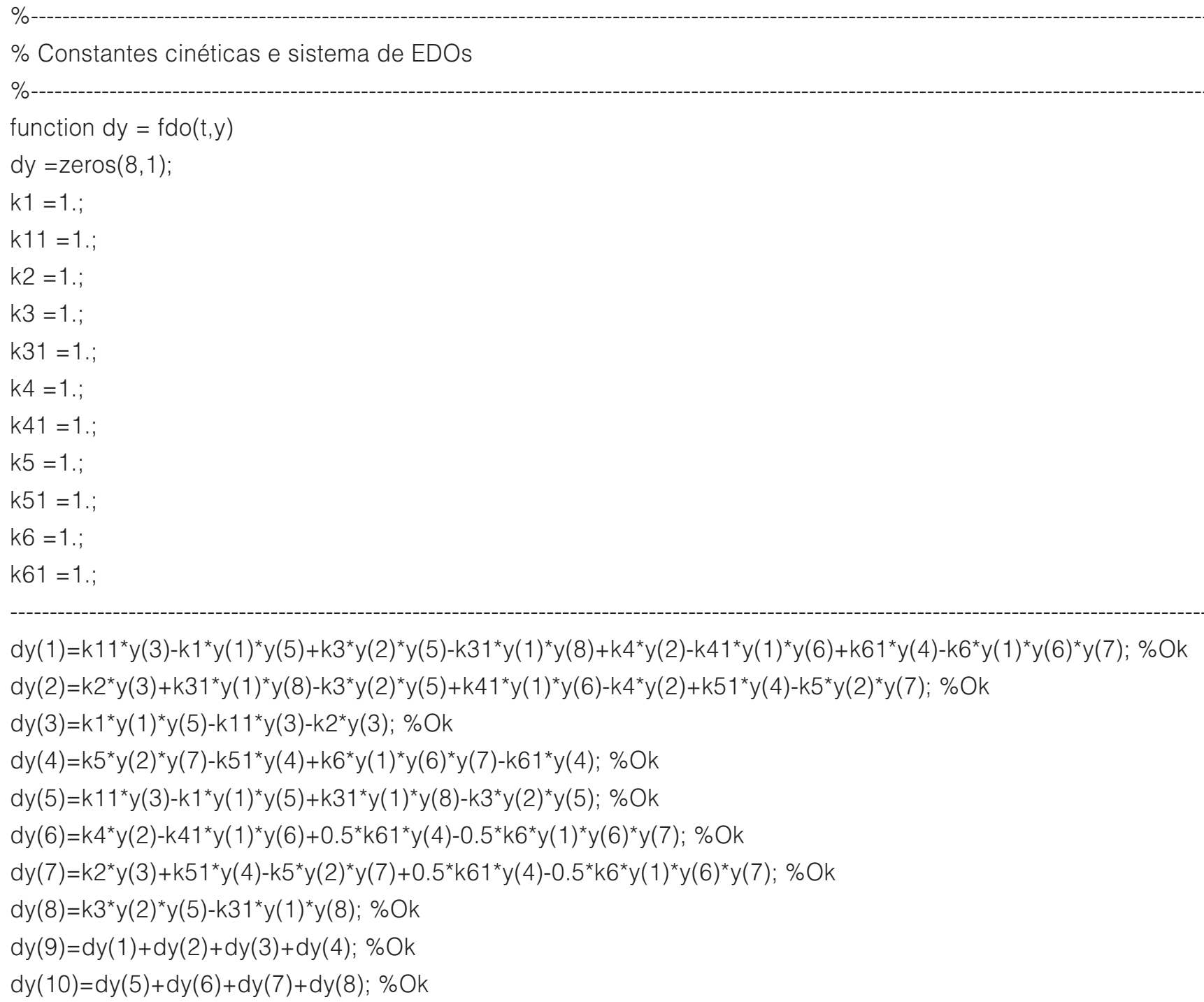

